\title{
THE INITIAL MASS FUNCTION : FROM SALPETER 1955 TO 2005
}

\author{
Gilles Chabrier \\ Ecole Normale Supérieure de Lyon, CRAL, Lyon, France \\ chabrier@ens-lyon.fr
}

\begin{abstract}
Fifty years after Ed Salpeter's seminal paper, tremendous progress both on the observational and theoretical sides allow a fairly accurate determination of the Galactic IMF not only down to the hydrogen-burning limit but into the brown dwarf domain. The present review includes the most recent observations of low-mass stars and brown dwarfs to determine this IMF and the related Galactic mass budget. The IMF definitely exhibits a similar behaviour in various environments, disk, young and globular clusters, spheroid. Small scale dissipation of large scale compressible MHD turbulence seems to be the underlying triggering mechanism for star formation. Modern simulations of compressible MHD turbulence yield an IMF consistent with the one derived from observations.
\end{abstract}

\section{Introduction}

The determination of the stellar initial mass function (IMF) is one of the holly grails of astrophysics. The IMF determines the baryonic content, the chemical enrichment and the evolution of galaxies, and thus the universe's light and baryonic matter evolution. The IMF provides also an essential diagnostic to understand the formation of stellar and substellar objects. In this review, we outline the current determinations of the IMF in different galactic environments, measuring the progress accomplished since Salpeter (1995) seminal paper 50 years ago. We also examine this IMF in the context of modern theories of star formation. A more complete review can be found in Chabrier (2003a) but very recent results are included in the present paper.

\section{Mass-magnitude relations}

Apart from binaries of which the mass can be determined eventually by use of Kepler's third law, the determination of the MF relies on the 
transformation of the observed luminosity function ( $\mathrm{LF}$ ), $\Phi=d N / d M$, i.e. the number of stars $N$ per absolute magnitude interval $d M$. This transformation involves the derivative of a mass-luminosity relationship, for a given age $\tau$, or preferentially of a mass-magnitude relationship $(\mathrm{MMR}), \frac{d n}{d m}(m)_{\tau}=\left(\frac{d n}{d M_{\lambda}(m)}\right) \times\left(\frac{d m}{d M_{\lambda}(m)}\right)_{\tau}^{-1}$, which applies directly in the observed magnitude $M_{\lambda}$ and avoids the use of often ill-determined bolometric and $T_{\text {eff }}$-color corrections.

Figure 1 displays the comparison of the Andersen (1991) and Ségransan et al. (2003) data in the V band with different theoretical MMRs, namely the parametrizations of Kroupa, Tout \& Gilmore (1993) (KTG), Reid et al. (2002) for $\mathrm{M}_{V}<9$ complemented by Delfosse et al. (2000) above this limit and the models of Baraffe et al. (1998) (BCAH) for two isochrones. The KTG MMR gives an excellent parametrization of the data over the entire sample but fails to reproduce the flattening of the MMR near the low-mass end, which arises from the onset of degeneracy near the bottom of the main sequence (MS), yielding too steep a slope. The Delfosse et al. (2000) parametrization, by construction, reproduces the data in the $\mathrm{M}_{V}=9-17$ range. For $\mathrm{M}_{V}<9$, however, the parametrization of Reid et al. (2002) misses a few data, but more importantly does not yield the correct magnitude of the Sun for its age. The BCAH models give an excellent representation for $m \gtrsim 0.4 \mathrm{M}_{\odot}$. Age effects due to stellar evolution start playing a role above $m \sim 0.8 \mathrm{M}_{\odot}$, where the bulk of the data is best reproduced for an age $1 \mathrm{Gyr}$, which is consistent with a stellar population belonging to the young disk $(h<100 \mathrm{pc})$. Below $m \sim 0.4 \mathrm{M}_{\odot}$, the BCAH MMR clearly differs from the Delfosse et al. (2000) one. Since we know that the BCAH models overestimate the flux in the Vband, due to still incomplete molecular opacities, we use the Delfosse et al. (2000) parametrization in this domain. The difference yields a maximum $\sim 16 \%$ discrepancy in the mass determination near $\mathrm{M}_{V} \sim 13$. Overall, the general agreement can be considered as very good, and the inferred error in the derived MF is smaller than the observational error bars in the LF. The striking result is the amazing agreement between the theoretical predictions and the data in the K-band (Figure 2), a more appropriate band for low-mass star (LMS) detections.

\section{The disk and young cluster mass function}

\subsection{The disk mass function}

A V-band nearby LF $\Phi_{V}$ can be derived by combining Hipparcos parallax data (ESA 1997), which are essentially complete for $\mathrm{M}_{V}<$ 12 at $r_{\text {comp }}=10 \mathrm{pc}$, and the sample of nearby stars with ground-based parallaxes for $\mathrm{M}_{V}>12$ with $r_{\text {comp }}=5.2 \mathrm{pc}$ (Dahn et al. 1986). Such 


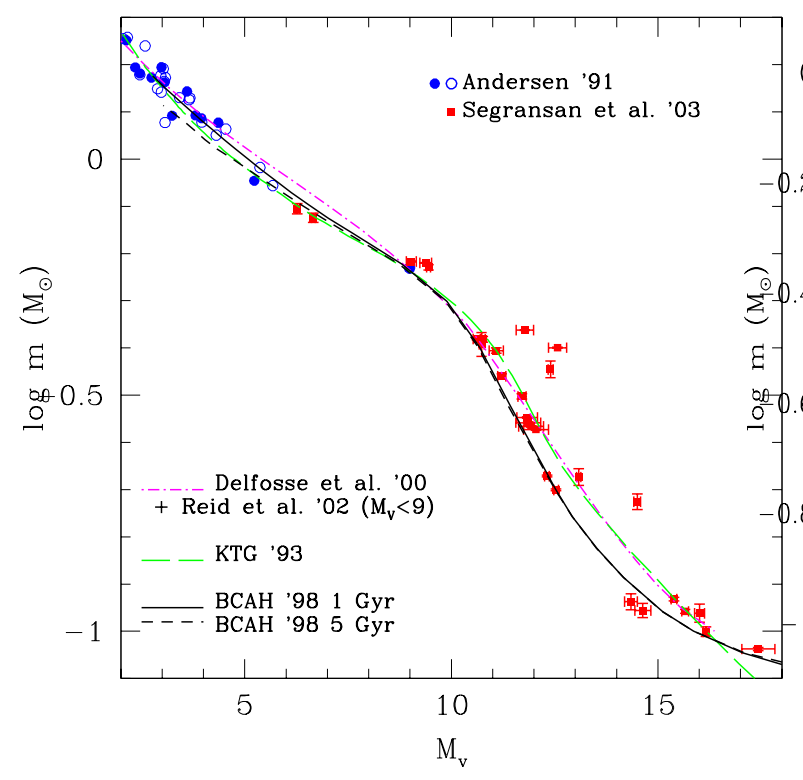

Figure 1. Comparison of the observed and theoretical $\mathrm{m}-\mathrm{M}_{V}$ relation.

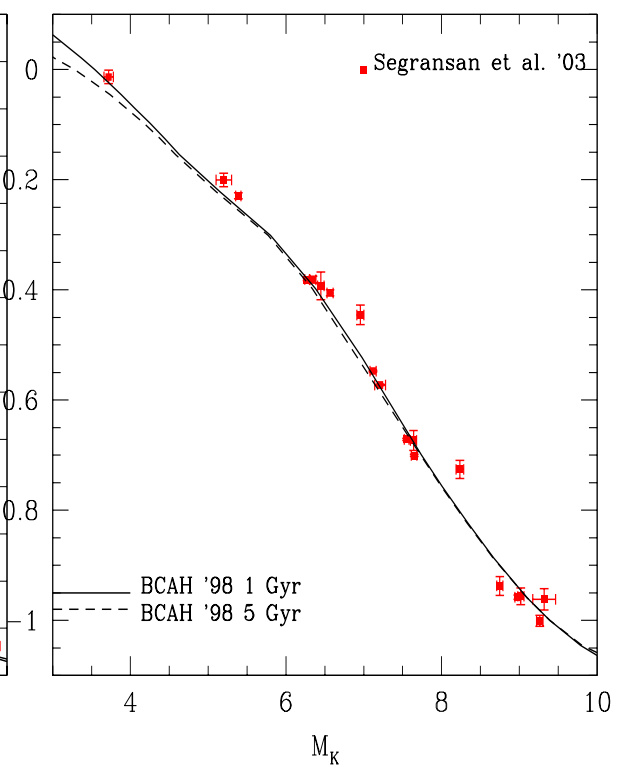

Figure 2. Same for the $\mathrm{m}-\mathrm{M}_{K}$ relation.

a sample has been reconsidered recently by Reid et al. (2002), and extended to $\mathrm{r}=8 \mathrm{pc}$ by Reid et al. (2004). The revised sample agrees within $\sim 1 \sigma$ with the previous one, except for $\mathrm{M}_{V} \gtrsim 14$, where the 2 LFs differ at the $\sim 2 \sigma$ limit. The 5 -pc LF was also obtained in the $\mathrm{H}$ and $\mathrm{K}$ bands by Henry \& McCarthy (1990).

The IMFs, $\xi(\log m)=\frac{d n}{d \log m}$, derived from the $\Phi_{V}$ and $\Phi_{K}$ LFs are portrayed in Figure 3 below $1 \mathrm{M}_{\odot}$. Superposed to the determinations is the following analytical parametrization (in $\left(\log \mathrm{M}_{\odot}\right)^{-1} \mathrm{pc}^{-3}$ ):

$$
\begin{aligned}
& \xi(\log m)=0.093 \times \exp \left\{-\frac{(\log m-\log 0.2)^{2}}{2 \times(0.55)^{2}}\right\}, m \leq 1 \mathrm{M}_{\odot} \\
& =0.041 m^{-1.35 \pm 0.3} \quad, m \geq 1 \mathrm{M}_{\odot}
\end{aligned}
$$

This IMF differs a bit from the one derived in Chabrier (2003a) since it is based on the revised 8-pc $\Phi_{V}$. The difference at the low-mass end between the two parametrizations reflects the present uncertainty at the faint end of the disk LF, near the H-burning limit (spectral types $\gtrsim$ M5). Note that the field IMF is also representative of the bulge IMF (triangles), derived from the LF of Zoccali et al. (2000). 


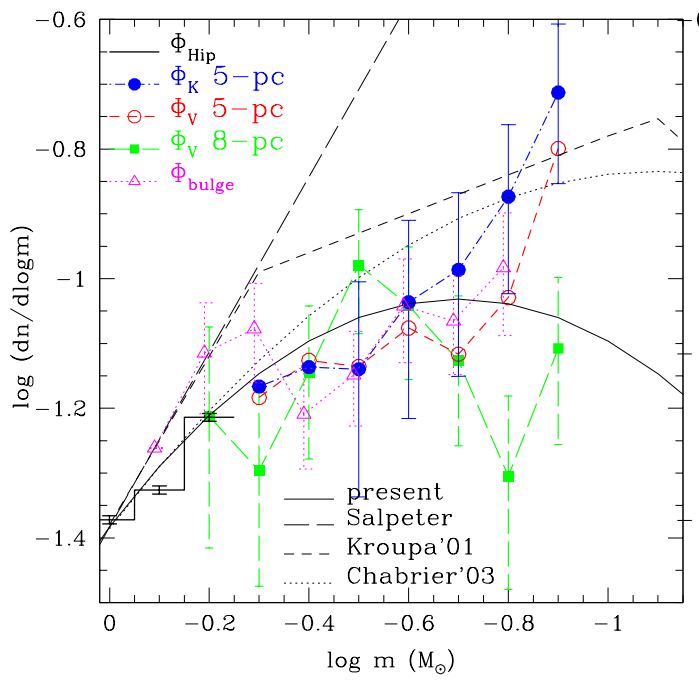

Figure 3. Disk IMF for individual objects.

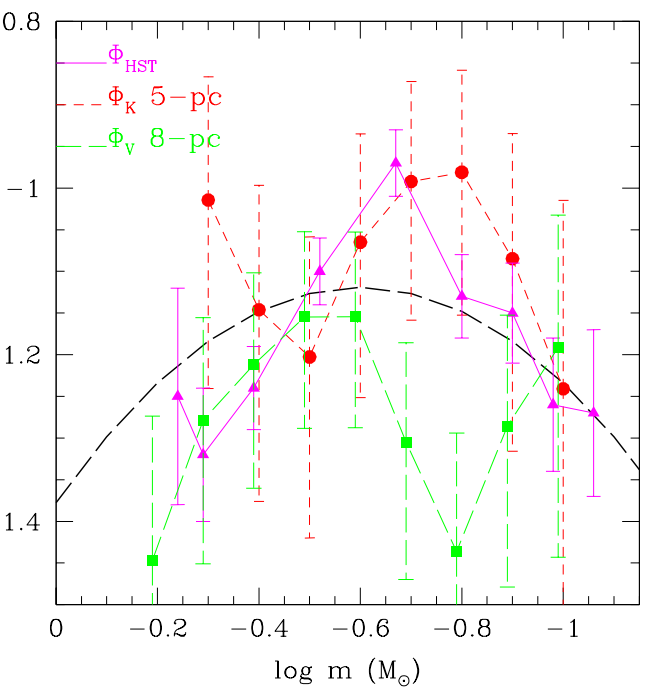

Figure 4. Disk IMF for unresolved systems.

A fundamental advantage of the nearby LF is the identification of stellar companions. It is thus possible to merge the resolved objects into multiple systems, to calculate the magnitude of the systems and then derive the system IMF. The following parametrization, slightly different from the one derived in Chabrier (2003a) is displayed in Figure 2 (normalyzed as eqn.(1) at $1 \mathrm{M}_{\odot}$, where all systems are resolved):

$$
\xi(\log m)=0.076 \times \exp \left\{-\frac{(\log m-\log 0.25)^{2}}{2 \times 0.55^{2}}\right\}, m \leq 1 \mathrm{M}_{\odot}
$$

As shown by Chabrier (2003b) and seen on the figure, this system IMF is in excellent agreement with the MF derived from the revised HST phometric LF (Zheng et al. 2001), showing that the discrepancy between the MF derived from the nearby LF and the one derived from the HST stemmed primarily from unresolved companions in the HST field of view.

The brown dwarf regime.

Many brown dwarfs (BD) have now been identified down to a few jupiter masses in the Galactic field with the DENIS, 2MASS and SDSS surveys. Since by definition BDs never reach thermal equilibrium and keep fading with time, comparison of the predicted BD LFs, based on a given IMF, with observations requires to take into account time (i.e. 


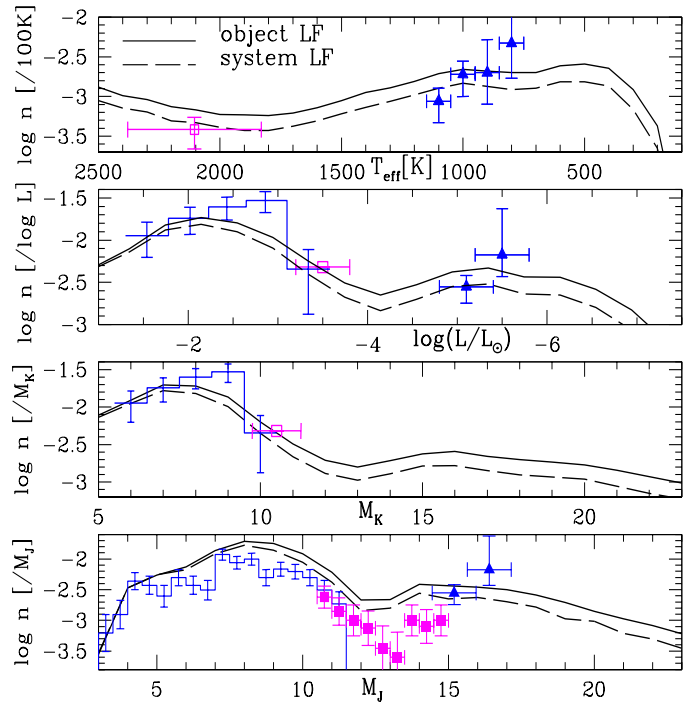

Figure 5. Comparison of the observed and theoretical LMS, L-dwarf (ם) and T-dwarf ( $\mathbf{\Delta})$ distributions.

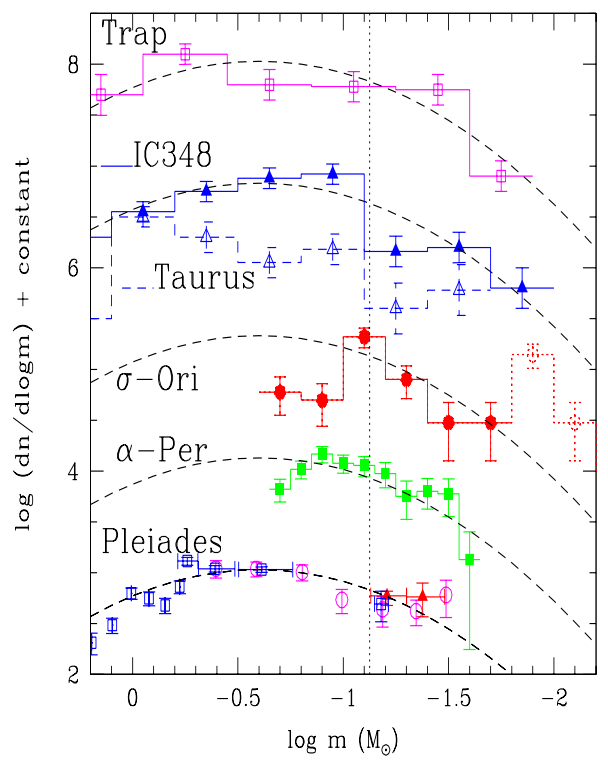

Figure 6. IMF for young clusters. Dash-line: field system IMF (2). Vertical dotted line: H-burning limit.

formation rate) and mass (i.e. IMF) probability distributions. In the present review, we proceed slightly differently from the calculations of Chabrier (2003a). We start from the system IMF (2) and we include a probability distribution for the binary frequency which decreases with mass. Indeed, various surveys now show that the binary fraction $X_{b i n}$ (and orbital separation) decreases with mass, varying from $X_{b i n} \approx 60 \%$ for $\mathrm{G}$ and K-stars to $\approx 40 \%$ for early (M0-M4) M-dwarfs, to $\approx 20 \%$ for later $\mathrm{M}$ and L-dwarfs, correcting for undetected short period binaries, to $\approx 10 \%$ for T-dwarfs.

Figure 5 displays the calculated BD density distributions as a function of $T_{\text {eff }}, L, \mathrm{M}_{K}$ and $\mathrm{M}_{J}$, based on the BD cooling models developed in the Lyon group, and the most recent estimated LMS and BD densities (Gizis et al. 2000, Burgasser 2001, Cruz 2004 ${ }^{1}$ ). The dash-line displays the distributions obtained with the system IMF (2) while the solid line corresponds to the IMF (1) for individual objects. As mentioned above, the distributions obtained with this latter IMF are consistent

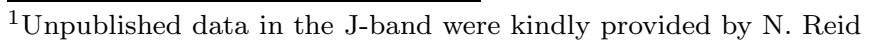


with a binary frequency decreasing from $\sim 50 \%$ to $\sim 20 \%$. The agreement between the theoretical calculations and the observations is very satisfactory, keeping in mind the remaining uncertainties in BD cooling theory and in accurate determinations of the observed BD $T_{\text {eff }}, \mathrm{M}_{b o l}$ and number densities. The predicted dip around $\mathrm{M}_{J} \sim \mathrm{M}_{K} \sim 13, \mathrm{M}_{b o l} \sim 15$ (Chabrier (2003a)) is confirmed by the recent L-dwarf observations.

\subsection{The young cluster mass function}

Figure 6 displays the MFs derived from the observed LFs of several young clusters, with ages ranging from $\sim 5 \mathrm{Myr}$ to $\sim 150 \mathrm{Myr}$, down to the substellar domain (see references in Chabrier (2003a)). Note that some of the faintest objects in $\sigma$-Or have been shown recently being field star contamination (McGovern et al. 2004, Burgasser et al. 2004). We used the MMRs from BCAH for the appropriate age in the appropriate observational filters. Accuracy of the BCAH models for young clusters has been examined carefully by Luhman et al. (2003). These observational surveys do not resolve multiple systems, so the derived MFs reflect the system MFs. Superposed to these IMFs is the field system IMF (2). Figure 6 clearly points to a similar underlying IMF between young clusters and the Galactic field, except for the significantly less dense Taurus cluster.

\section{The globular cluster and spheroid mass function}

Globular clusters provide a particularly interesting test-bed to investigate the stellar MF. They provide a homogeneous sample of MS stars with the same age, chemical composition and reddening, their distance is relatively well determined, allowing straightforward determinations of the stellar LF. From the theoretical point of view, the Baraffe et al. (1997) evolutionary models accurately reproduce the observed colormagnitude diagrams of various clusters with metallicity $[\mathrm{M} / \mathrm{H}] \leq-1.0$ both in optical and infrared colors, down to the bottom of the main sequence, with the limitations in the optical mentioned in $\S 2$ for more metal-rich clusters. As mentioned above, however, the consequences of this shortcoming on the determination of the MF remain modest. The IMFs derived by Chabrier (2003a) for several globular clusters, from the LFs observed with the HST, corrected for dynamical evolution, by Paresce \& DeMarchi (2000) are displayed in Fig.7. Superposed to the derived IMFs is the spheroid IMF given by eqn.(20) of Chabrier (2003a) (short-dash line), with the characteristic mass shifted by 1 to 2 $\sigma$ 's $\left(m_{c}=0.22\right.$ to $\left.0.32 \mathrm{M}_{\odot}\right)$, similar to the IMF derived by Paresce \& 
DeMarchi (2000). The disk IMF (2) is also superposed for comparison. We note the relative similarity of the these two IMFs, but a defficiency of very-low-mass objects, including BDs, in the cluster IMFs.

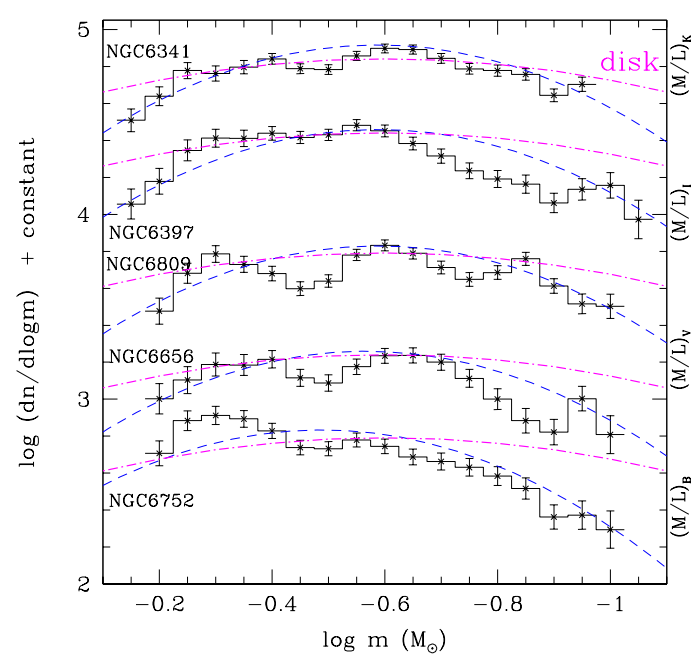

Figure 7. IMF for several globular clusters. Dot-dash line: disk system $\operatorname{IMF}(2)$

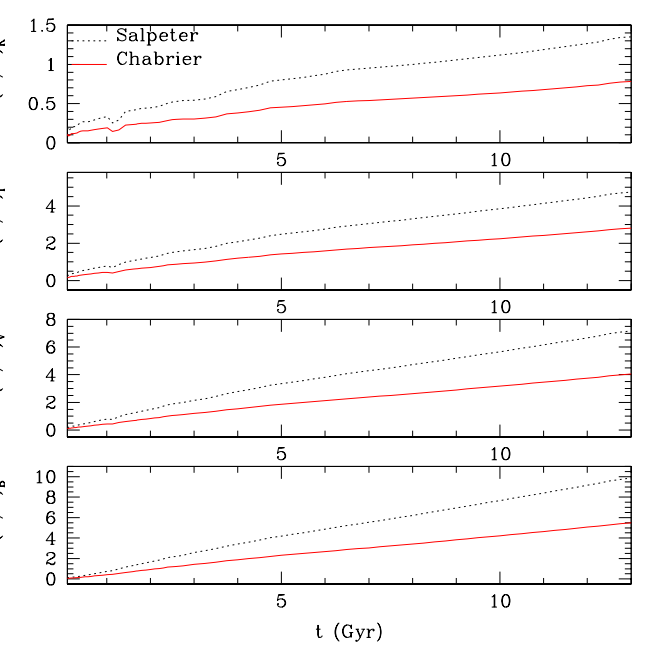

Figure 8. Mass-to-light ratio for single stellar population in optical and NIR bands.

\section{Galactic implications : mass budget and mass to light ratio}

Integrating the IMF (1) yields the stellar and brown dwarf numberand mass-densities given in Table I, and the relative contributions $\mathcal{N}=$ $N(\Delta m) / N_{\text {tot }}$ and $\mathcal{M}=M(\Delta m) / M_{\text {tot }}$, where $N$ and $M$ denote respectively the number and mass of objects in the mass range $\Delta m$. Adding up remnant densities (Chabrier (2003a)) yields the stellar+substellar contributions to the Galactic mass budget. These new determinations give a BD-to-star number ratio of $n_{B D} / n_{\star} \sim 1 / 3$.

Figure 8 compares the M/L ratio obtained with the present IMF (1) and with the Salpeter IMF (see also Bruzual \& Charlot 2003). The present IMF yields $\mathrm{M} / \mathrm{L}$ ratios in good agreement with observations and with expectations from CDM hierarchical simulations of spiral disk galaxies or from dynamical arguments (e.g. from Tully-Ficher relation), whereas a Salpeter IMF yields too large values (Portinari et al. 2004). 
Table 1. Present day stellar and brown dwarf Galactic budget ${ }^{a, b}$.

\begin{tabular}{|c|c|c|c|c|}
\hline Parameter & & Disk & Spheroid & Dark halo \\
\hline$n_{B D}$ & & $2.6 \times 10^{-2}$ & $3.5 \times 10^{-5}$ & \\
\hline$\rho_{B D}$ & & $1.0 \times 10^{-3}$ & $\lesssim 2.3 \times 10^{-6}$ & \\
\hline$n_{\star}$ & & $(9.3 \pm 2) \times 10^{-2}$ & $\leq(\widetilde{2.4} \pm 0.1) \times 10^{-4}$ & \\
\hline$\rho_{\star}$ & & $(3.4 \pm 0.3) \times 10^{-2}$ & $\leq(6.6 \pm 0.7) \times 10^{-5}$ & $\ll 10^{-5}$ \\
\hline$n_{r e m}$ & & $(0.7 \pm 0.1) \times 10^{-2}$ & $\leq(2.7 \pm 1.2) \times 10^{-5}$ & \\
\hline$\rho_{\text {rem }}$ & & $(0.6 \pm 0.1) \times 10^{-2}$ & $\leq(1.8 \pm 0.8) \times 10^{-5}$ & $<10^{-4}$ \\
\hline$n_{\text {tot }}$ & & $0.13 \pm 0.03$ & $\leq 3.0 \times 10^{-4}$ & \\
\hline$\rho_{\text {tot }}$ & & $(4.1 \pm 0.3) \times 10^{-2}$ & $\leq(9.4 \pm 1.0) \times 10^{-5}$ & $<10^{-4}$ \\
\hline BD: & $\overline{\mathcal{N}} ; \mathcal{M}$ & $0.20 ; 0.02$ & $0.10 ; 0.03$ & \\
\hline $\operatorname{LMS}\left(\leq 1 \mathrm{M}_{\odot}\right):$ & $\mathcal{N} ; \mathcal{M}$ & $0.71 ; 0.68$ & $0.80 ; 0.77$ & \\
\hline $\operatorname{IMS}\left(1-9 \mathrm{M}_{\odot}\right):$ & $\mathcal{N} ; \mathcal{M}$ & $0.03 ; 0.15$ & $0 . ; 0$. & \\
\hline WD+NS: & $\mathcal{N} ; \mathcal{M}$ & $0.06 ; 0.15$ & $0.10 ; 0.20$ & \\
\hline
\end{tabular}

${ }^{a}$ The number densities $n$ are in $\left[\mathrm{pc}^{-3}\right]$, the mass densities $\rho$ are in $\left[\mathrm{M}_{\odot} \mathrm{pc}^{-3}\right]$.

\section{Star formation theory}

Although we are still far from a general paradigm for star formation, some general properties can be considered as robust : (1) star formation extends well below the H-burning limit, (2) the shape of the IMF seems to be very similar in very diverse environments, (3) star formation is a rapid process, comparable to the dynamical timescale $\tau_{\text {dyn }}=(3 \pi / 32 G \rho)^{1 / 2} \approx 1-5 \times 10^{5}$ yr for typical star-forming molecular clouds, (4) the stellar IMF seems to be reminiscent of the prestellar core mass spectrum, suggesting that the IMF is already determined by the cloud clump mass distribution (see André and Myers, this conference), (5) on large scales, the spectral line widths in molecular clouds indicate supersonic, super-Alfvénic conditions. All these observations point to a common driving mechanism for star formation, namely turbulencedriven fragmentation. Figure 10 compares the mass spectrum obtained from MHD simulations done recently by Padoan \& Nordlund (2002) and Li et al. (2004) with the system IMF (2) (indeed, the prestellar cores correspond to multiple systems, which eventually will fragment further into individual objects). Although such comparisons must be considered with due caution before drawing any conclusion, the agreement between the simulations and the IMF representative of the field is amazing. Note that such hydrodynamical simulations form BDs in adequat numbers from the same fragmentation mechanism as for star formation. Various observations of disk accreting BDs indeed show that BDs and stars 
form from the same underlying mechanism. Motivation for invoking the formation of BDs by ejection thus no longer seems to be necessary. Moreover, the recent observation of a wide binary BD (Luhman 2004) definitely contradicts the predictions of such a scenario as a dominant formation mechanism for BDs. This new picture thus combines turbulence, as the initial driving mechanism for fragmentation, and gravity, providing a natural explanation for a (scale free) power-law IMF above a critical mass, namely the mean thermal Jeans mass $\left\langle m_{J}\right\rangle$, and a lognormal distribution below, due to the fact that only the densest cores, exceeding the local Jeans mass, will collapse into bound objects. Note that in these simulations of supersonic turbulence, only a few percents of the total mass end up into the collapsing cores after one dynamical time, solving naturally the old high efficiency problem associated with turbulence-driven star formation. Recent similar simulations by Li \& Nakamura (2004), on the other hand, suggest enhanced ambipolar diffusion to occur through shock compression.

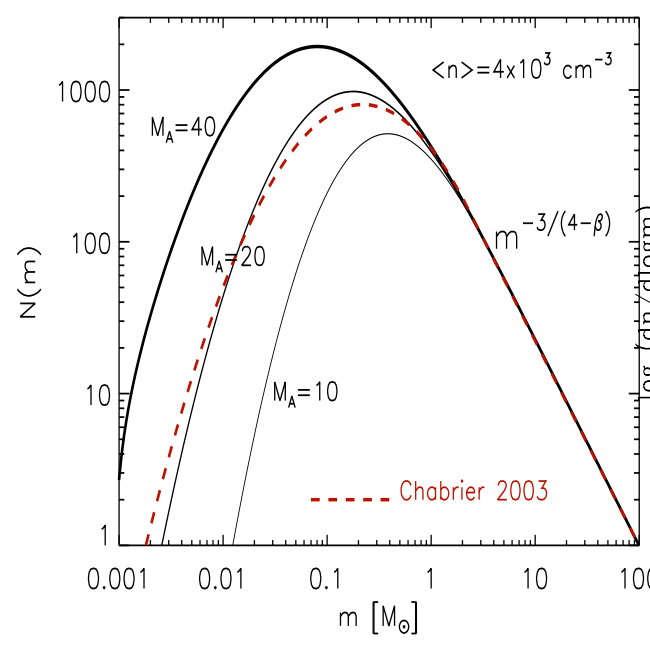

Figure 9. Comparison of the system IMF (2) with the one obtained by Padoan \& Nordlund (2002)

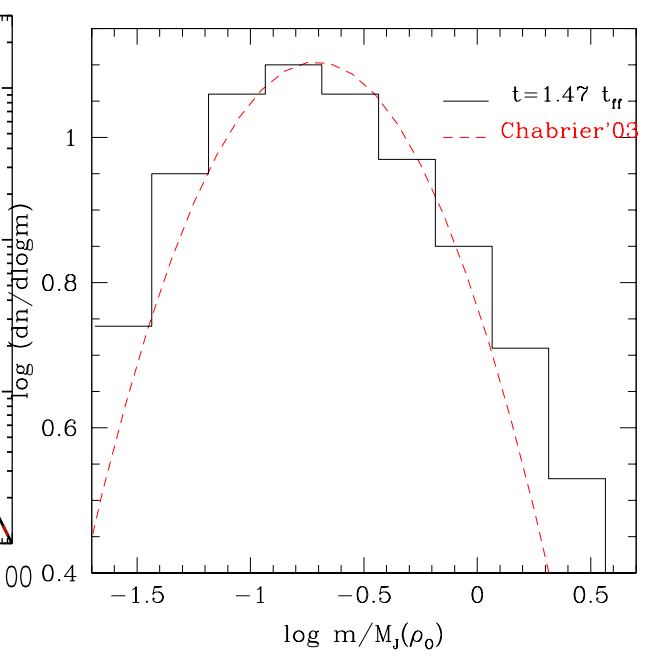

Figure 10. Same with the one obtained from MHD simulations by Li et al. (2004).

\section{Conclusion and perspective}

In this review, we have examined the most recent determinations of the Galactic stellar IMF. Thanks to tremendous progress both in observational techniques and in the theory of low-mass stars and brown 
dwarfs, the IMF can now be determined down to a few jupiter masses, two orders of magnitude below the $\sim 0.5 \mathrm{M}_{\odot}$ limit of accuracy of the Salpeter (1955) IMF. This IMF adequately reproduces various observational constraints, star and $\mathrm{BD}$ counts, binary frequencies, galactic mass-to-light ratios. It is well described by a Salpeter power-law above $\sim 1 \mathrm{M}_{\odot}$ rolling down into a lognormal form below this mass, with a characteristic mass around $0.2 \mathrm{M}_{\odot}$, although more data is needed to really nail down this issue. Only for nearly zero-metal environments in the early universe do we expect a significantly larger characteristic mass, due to the lack of efficient cooling mechanism in the cloud (see Bromm, this conference). The universality of the IMF in various environments, disk, young and globular clusters, spheroid, points to a universal triggering mechanism and a dominant cooling process for star formation. Small scale dissipation of large scale supersonic MHD turbulence provides an appealing solution for this mechanism.

\section{References}

Andersen, J., 1991, A\&ARv, 3, 91

Baraffe, I., Chabrier, G., Allard, F., \& Hauschildt, P., 1997, A\&A, 327, 1054

Baraffe, I., Chabrier, G., Allard, F., \& Hauschildt, P., 1998, A\&A, 337, 403

Bruzual \& Charlot 2003, MNRAS, 344, 1000

Burgasser, A., 2001, PhD thesis

Burgasser, A., et al., 2004, ApJ, 604, 827

Chabrier, G., 2003a, PASP, 115, 763

Chabrier, G., 2003b, ApJ, 585, L133

Cruz, K., 2004, PhD thesis

Dahn, C.C, Liebert, J., Harrington, R.S, 1986, AJ, 91, 621

Delfosse, X., et al., 2000, A\&A, 364, 217

Gizis, J., et al. 2000, ApJ, 120, 1085

Henry, T.J., \& McCarthy, D.W., 1990, ApJ, 350, 334

Kroupa, P., 2001, MNRAS, 322, 231

Li, P.S.,Norman, M., Mac Low, M.-M., \& Heitsch, F., 2004, ApJ, 605, 800

Li, Z.-Y., \& Nakamura, F., 2004, ApJ, 609, L83

Luhman, K., 2003, ApJ, 593, 1093; 2004, astro-ph/0407344

McGovern, J., et al. 2004, ApJ, 6001020

Padoan, P., \& Nordlund, Å, 2002, ApJ, 576, 870; astro-ph/0205019

Reid, I.N., Gizis, J.E. \& Hawley, S.L., AJ, 2002, 124, 2721

Reid, I.N., et al., 2004, in preparation

Paresce, F., \& De Marchi, G., 2000, ApJ, 534, 870

Portinari, Sommer-Larsen \& Tantalo, 2004, MNRAS, 347, 691

Salpeter, E.E., 1995, ApJ, 121, 161

Ségransan, D., et al., 2004, IAU Symposium 211, Astr. Soc. Pacific, 2003, p. 413

Zheng, Z, Flynn, C., Gould, A., Bahcall, J.N., \& Salim, S., 2001, ApJ, 555, 393

Zoccali, M. et al., 2000, ApJ, 530, 418 